\title{
Socialisation religieuse, engagement militant et carrières professionnelles
}

\author{
Transfert de dispositions et travail de mise en \\ cohérence biographique
}

Gilles Descloux

\begin{abstract}
[Résumé] Cet article examine les incidences de l'engagement militant sur la carrière professionnelle. II repose sur une analyse dispositionnelle de l'engagement de deux individus ayant fréquenté des organisations catholiques qui, dans les années 1968, ont été traversées par un mouvement de politisation de l'engagement religieux. La manière dont ils sont amenés à endosser, après leur engagement, leur profession, remettant partiellement en cause certaines pratiques professionnelles, est largement redevable de leur socialisation religieuse et militante antérieure. Cependant, les contextes professionnels façonnent également, en retour, leurs dispositions et sollicitent un travail de mise en cohérence de leurs parcours biographiques.
\end{abstract}

Mots-clés : Carrière militante ; incidences biographiques ; engagement religieux ; analyse processuelle; sphère de vie ; ressources humaines.

[Abstract] This paper examines the consequences of activism on the professional career. Through a dispositional analysis of two individuals' involvement in catholic organizations, politically and religiously transformed during the 1968's, the paper focuses on the way they assumed their professional positions after this period of radical political critics. It demonstrates that how they embrace and apprehend their professional positions relies partly on dispositions internalized during their past political involvement. But the professional contexts also shaped in return their dispositions and lead them to revisit their biographical past in a cohesiveness way with their current position what Pierre Bourdieu calls "biographical illusion".

Keywords: Activist career; biographical consequences; religious involvement; processual analysis; life spheres; human resources.

Au printemps 1968, les étudiants et apprentis en Suisse prennent part au mouvement contestataire international pour exiger une transformation radicale des structures en place. Certains catholiques entrent dans ce mouvement à travers une politisation de l'engagement religieux qui les amène à endosser une posture critique à l'égard des institutions religieuses, politiques ou encore syndicales. Alors que cette période d'émulation contestataire s'évanouit au cours des années 1970, on pourrait penser que ces militants « se rangent » et empruntent des bifurcations professionnelles (Denave,

${ }^{1}$ Université de Lausanne, Institut d'études politiques, historiques et internationales (IEPHI) et Centre de recherche pour l'action politique de l'université de Lausanne (CRAPUL). 
2015) qui les amènent à exercer un travail « comme tout le monde ». On veut montrer ici que l'activité militante porte de profondes incidences sur la manière dont ils endossent leurs professions, c'est-à-dire s'approprient et s'accommodent des normes et exigences encadrant leur travail. L'article analyse dès lors les processus à travers lesquels la politisation de l'engagement religieux porte des incidences sur la manière dont ces individus investissent leurs rôles professionnels, mais aussi la façon dont les différents contextes de la carrière professionnelle façonnent en retour leurs dispositions et sollicitent un travail de mise en cohérence des différentes étapes de leur parcours biographique (Voegtli 2004).

Cette analyse s'inscrit dans la lignée des travaux qui pensent ensemble la politisation, le devenir militant et la sphère professionnelle (Sainsaulieu et Surdez, 2012), en veillant « à prendre les choses dans l'ordre dans lequel elles se présentent dans la réalité des expériences » (Lahire, 2011 : 11). L'analyse de ces processus biographiques repose sur l'étude approfondie de deux parcours de vie. On verra en quoi le processus amenant deux ex-militants à endosser leur profession comme ils le font est redevable d'un ensemble de propriétés et processus telles que l'origine sociale, la socialisation religieuse, ou encore les ressources acquises à travers leur engagement religieux, militant et professionnel. Leur comparaison permettra de faire ressortir des logiques que l'on retrouve chez l'ensemble des militants interviewés dans le cadre d'une recherche de doctorat portant sur les conséquences biographiques de l'engagement militants «à la gauche du Christ » en Suisse romande (N=32).

\section{Michel : de l'Ordre dominicain à l'éthique politique dans la coopération internationale}

Le premier cas que nous allons analyser est celui de Michel, né en 1943. Il grandit dans une famille catholique, d'un père médecin, gradé à l'armée, et d'une mère au foyer. Dans une région rurale encadrée par l'Église et dirigée par des notables catholiques, il assiste comme tous les enfants du village à la messe et aux activités paroissiales. Lorsqu'il a cinq ans, son père décède d'un accident survenu lors d'un entraînement militaire : « Cela m’a fortement marqué [...] c'était une douleur extrême », explique-t-il. La famille déménage alors dans une ville universitaire, sa mère étant soucieuse d'offrir "plus de chance pour sa fille et ses garçons », et pour fuir le statut de «la veuve éplorée pendant toute sa vie ». Dotée d'un diplôme d'employée de commerce sans expérience professionnelle, sa mère décroche un emploi qui subvient à peine aux besoins du foyer : "Alors qu'on était d'une famille bourgeoise, on a vécu souvent des fins de mois très difficiles ».

Dans ce nouvel environnement, Michel trouve sa principale source d'épanouissement dans le scoutisme, activité propice à l'acquisition de dispositions relatives à l'entraide et à la valorisation de l'aide aux autres, mais aussi dans les activités de la paroisse catholique du quartier. Ces activités qui vont de pair « avec un 
enracinement dans un univers associatif et communautaire» (Siméant, 2001: 62) constituent, à cette époque, des lieux de socialisation juvéniles importants, d'autant plus pour les catholiques de la « diaspora » en régions à forte majorité protestante. C'est dans ce cadre qu'il se confie et s'entretient régulièrement avec un aumônier dominicain de son lycée « qui m’avait pas mal impressionné » et qui joue un rôle décisif dans son orientation au séminaire. Alors que son cursus scolaire voue la plupart de ses camarades de classe aux écoles supérieures d’ingénieurs (« Beaucoup de mes collègues ont fait l'EPFL [i.e. institution prestigieuse de formation des ingénieurs]»), Michel choisit la théologie. Plutôt introverti et peu à l'aise dans les situations qui s'écartent trop de ce cadre de sociabilité familier, il trouve chez les Dominicains un lieu de repli et d'encadrement rassurant : «J'avais une recherche... un petit peu une... une peur de la vie. J'avais aussi envie d'avoir une vie rythmée, assez méditative ». Durant sept ans, Michel grandit dans la ritualité caractéristique des ordres religieux instituant une séparation stricte d'avec la société, et qui se concentre autour de trois disciplines théologiques principales, à savoir le dogme, la morale et le droit canon (Planzi, 2011 : 332). Ce cadre propice à l'intériorisation du régime de vérité catholique (Lagroye, 2006) en dehors de toute distraction et d'urgence économique renforce son appétence pour le travail intellectuel : «Du côté dominicain, on a quand même une tradition de valorisation des études ». Une fois son séminaire achevé et couronné de l'ordination, Michel, comme bon nombre de ses coreligionnaires, poursuit son cursus en thèse.

Il va éprouver alors une forte insatisfaction à l'égard des structures rigides et autoritaires de l'institution catholique, à l'instar de plusieurs de ses coreligionnaires. Le Concile Vatican II (1963-1967) promulgue ses encycliques incitant à l'action des chrétiens dans le monde et à leur engagement pour une plus grande justice sociale. À la fin des années 1960, des prêtres et aumôniers pour qui la division des chrétiens pour des raisons doctrinales ne fait plus sens s'approprient ce renouveau théologique en faveur d'un engagement commun et solidaire au côté des plus démunis. Confrontés aux transformations de leur environnement social, toute une génération de prêtres et frères religieux vivent les anciennes formes du sacerdoce comme une régression vers un catholicisme abstrait et désengagé. C'est notamment le cas chez certains frères étudiants pour qui l'ordre dominicain transmet « certes une profonde formation culturelle et spirituelle, mais peut-être pas assez humaine et pastorale »(Planzi, 2011 : 333). Conformément aux appels répétés du concile et du mouvement œcuménique pour la justice et la fraternité « au nom du Christ » (Fouilloux, 2000), ces religieux valorisent de nouvelles formes d'engagement et de réflexion théologique susceptibles de fournir un support à l'action, favorisant les échanges avec les milieux protestants. Michel fréquente régulièrement ces derniers, à l'occasion des premiers rassemblements œcuméniques, mais aussi dans le cadre de sa thèse et à travers ses lectures :

Il y a eu pas mal de recherches musicales et artistiques, de textes aussi, Sullivan, Michel Quoist. [...] Un certain nombre d'auteurs qui tentaient de former des prières ou l'expression d'un questionnement [...] en langage vernaculaire, en langage non codé si vous voulez. C'était quelque chose d'important. 
Admiratif de ces prêtres capables de sortir de leur isolement religieux pour investir la réflexion socio-politique, Michel s'efforce d'adopter une analyse sociohistorique des énoncés théologiques dans le cadre de sa thèse : « J'avais choisi un commentaire de Calvin. C'était un petit peu pour me discipliner parce j'avais une tendance à avoir une réflexion relativement abstraite ». Ses recherches le poussent à réfléchir aux conditions sociales de production et d'interprétation des textes bibliques et commentaires théologiques. Cette posture l'amène à se détacher du rapport doxique à l'institution religieuse en interrogeant ses présupposés tacites : «Quand j'étais aux études, j’ai pu voir toute une série de gens qui étaient dans un langage qui... comment dire... qui était autoperformant. Vous commencez la phrase et vous savez où elle se termine ». Les propositions ecclésiologiques du dominicain Yves Congar ${ }^{2}$, valorisant l'autonomie du croyant et remettant en cause la hiérarchie entre clercs et laïcs, nourrissent alors ses questionnements.

Parallèlement à sa thèse, Michel est confronté à de nouvelles exigences lorsqu'il est nommé aumônier à temps partiel dans une université remuée par les événements de 1968. Il y éprouve une grande difficulté à débattre et échanger avec des étudiants mobilisant des analyses marxistes dont la portée explicative convainc pourtant de nombreux jeunes catholiques à rejoindre les groupes gauchistes, et vit mal le décalage entre leurs préoccupations et ce pour quoi le séminaire l'a préparé:

Je me rendais compte que soit il y avait peu d'intérêt pour la dimension spirituelle, ou alors... oui, je n'arrivais pas à trouver... je n'étais pas l'aise avec le langage de l'Église, ce langage formaté, et je n'arrivais pas à formuler une alternative. J'arrivais de temps en temps dans des circonstances, lors d'une célébration d'un mariage, où j'arrivais à formuler des choses de façon plus originale, et plus satisfaisante pour moi, mais publiquement c'était plus difficile.

C'est dans ce contexte qu'il se lie d'amitié avec l'équipe des aumôniers protestants et catholiques engagés dans la mouvance conciliaire et œcuménique imprégnée du gauchisme des années 1968. Il sympathise en particulier avec un coreligionnaire "politiquement plus à gauche » et «anti-establishment » : «'étais plus... un petit peu accommodant, en essayant de balancer un peu les expériences. Lui voyait très vite... les politiciens simplement s'accaparer du pouvoir ». Au printemps 1969, ces aumôniers mettent sur pied un rassemblement œcuménique hebdomadaire pour les jeunes valorisant l'esprit communautaire, la participation de chacun et l'expression plus spontanée d'une foi questionnée à partir des enjeux sociopolitiques du présent :

\footnotetext{
${ }^{2}$ Enseignant au Saulchoir dominicain près d'Evry (Paris) sanctionné en 1954 pour ses audaces théologiques qui ouvrent le dialogue avec le marxisme, le théologien dominicain français Yves Congar, victime de censures et tracas sous Pie XII pour «ses accointances réelles ou supposées avec les prêtres-ouvriers » (Pelletier, 2002, 20), fait autorité pour de nombreux catholiques qui s'inscrivent dans la mouvance des «chrétiens de gauche » (cf. Pelletier et Schlegel, 2012), tout en gardant une certaine distance critique à l'égard des revues et théologiens proches de la théologie de la révolution.
} 
Ça, c'était un choc très positif. Et comme on avait beaucoup de discussions entre nous, une matinée chaque semaine, des questions d'interprétation des textes bibliques... j'étais plus à l'aise avec l'approche... comment dire... avec l'approche protestante, où il n'y a pas une approche autoritaire qui est fixée, mais une confrontation des approches.

Le succès inattendu d'un rassemblement attirant des centaines de jeunes catholiques et protestants, qui décident de partager ensemble l'intercommunion, est perçu comme une audace inacceptable par les autorités religieuses. Elles reprennent sévèrement $\mathrm{Mi}$ chel et ses collègues et mettent définitivement un terme à l'expérience. Cette sanction assoit Michel dans son sentiment d'avoir affaire à une Église renfermée sur elle-même : Les Églises étaient dans leur moule, dans leur formatage idéologique, je dirais, de langage aussi. Là, je pense qu'il y a eu quelque chose de fort. Ça a été pour moi le signal de l'arrogance de l'autorité ecclésiastique. [...] C'était une espèce de schizophrénie... moi j'essayais de repenser, de redire ma foi, ou ce qu'il me reste, dans une postmodernité.

À ce désajustement en succèdent d'autres. Alors que l'objection de conscience et l'antimilitarisme atteignent leur zénith, il emménage en communauté avec des militants pacifistes protestants avec qui il s'est lié d'amitié dans le cadre du rassemblement œcuménique. Dans ce contexte propice à la contestation politique de l'armée dont la violence fait écho au décès de son père, Michel appose sa signature sur la «liste des 32 » prêtres et pasteurs ${ }^{3}$ renonçant à payer leur taxe militaire - un geste qui provoque « des discussions très dures " avec ses supérieurs. Finalement, devant les démissions en chaîne de prêtres dominicains, il décide à son tour de quitter l'ordre :

C'est vrai qu'il y a aussi d'autres amis qui ont quitté au même moment, et je me suis senti un petit peu le dernier des Mohicans. Je pense que cela a aussi joué un rôle. Mais c'est principalement la résistance sur... l'arrogance du système, l'arrogance de l'autorité ecclésiastique, et sa prétention.

Suite à sa démission, il finance les cinq dernières années de sa thèse en dispensant à temps partiel des cours d'éthique et de théorie de la communication dans une école formant les premiers professionnels de l'encadrement socioculturel. En passant de l'analyse des énoncés théologiques aux questions éthiques, il parvient temporairement à faire valoir ses ressources et compétences dans le domaine séculier de l'action sociale. Il y rencontre sa future épouse, une femme « athée et anarchiste » à qui il attribue « un sens terrible pour détecter les petites manœuvres [...] et la prétention des grands ».

Michel entre alors dans une période d'incertitude professionnelle, marquée par quelques mois de chômage, durant laquelle il rédige, par intérêt personnel, un

\footnotetext{
${ }^{3}$ En 1972, une lettre publique signée par 32 prêtres et pasteurs de toute la Suisse romande exprime leur « refus collectif à la défense nationale » en réponse à l'appel des « déclarations de nos églises » (Assemblée EFcuménique des Églises à Uppsala, encyclique Populorum Progressio, Conférence Interconfessionnelle Suisse-Tiers-Monde). Soutenue par quarante-trois ecclésiastiques de toute la Suisse francophone, la déclaration dénonce le soutien de l'institution militaire aux seuls intérêts économiques, justifiant ainsi leur refus de s'acquitter de la taxe militaire.
} 
répertoire bibliographique des périodiques missionnaires, témoignant d'un certain attachement pour un engagement religieux orienté vers l'Outre-mer. Une série de contingences l'amènent ensuite, cinq ans après sa démission de l'ordre dominicain, à décrocher un poste auprès de la Confédération en tant que chef de projet en politique de développement. Lors d'un remplacement temporaire dans une faculté de théologie dans un pays d'Afrique du sud-ouest, il rencontre en effet un collaborateur de la Direction du développement et de la coopération (DDC) qui, dès son retour en Suisse, le recommande auprès de la Confédération dans le cadre d'un nouveau projet :

C'était un projet construit par la Banque mondiale, [un pays africain] et la Suisse, avec... comment dire... un volet d'ingénierie technique très lourd, et un volet d'ingénierie sociale minimaliste. II fallait déplacer des gens, il y avait tout un roblème de participation de quartier. C'était un quartier de 120000 personnes, hein. Et donc il voulait quelqu'un qui avait un feeling.

Michel doute toutefois de sa capacité à remplir cette tâche et ressent une forte insécurité. Il affirme avoir eu « très peur, parce que je me retrouve dans un tout autre métier, une tout autre activité, qui va durer cinq ans, qui est un travail dans un bidonville». En même temps, cette opportunité professionnelle lui fournit un espace de réalisation de soi dans la mesure où elle entre en accord avec les incitations d'un milieu catholique marqué par un concile appelant à s'engager auprès des plus démunis, en particulier dans les pays du «tiers-monde ». À ce stade de son parcours, Michel intègre une activité professionnelle pour laquelle il semble dépourvu d'expérience et de compétences spécifiques. Leur acquisition progressive va s'accompagner de l'activation de certaines dispositions intériorisées durant sa socialisation religieuse et militante. Envoyé sur le terrain en Afrique, Michel est chargé de coordonner un projet consistant à réaménager un territoire occupé par un bidonville. Malgré la découverte difficile de l'extrême misère, il vit une première expérience de terrain positive (" on a eu toute une série de liens joyeux ») et acquiert des savoirs ajustés à ses attentes : «Ça été très bien parce que j'ai mieux compris l'importance de l'économie, mais aussi le degré de liberté que cela [certains dispositifs économiques] peut amener pour être aussi innovant ». Cet engagement professionnel l'amène à mettre en œuvre des procédures édictées par des instances, telles que la Banque mondiale ou des gouvernements nationaux, dominées par des économistes et ingénieurs focalisés sur le développement technique et l'analyse de facteurs macro-économiques.

Dans le cadre de cette activité, Michel se distingue par une sensibilité particulière contre l'arbitraire des arrangements quotidiens défavorable à certains groupes sociaux, disposition caractéristique et transversale aux participants des mouvements contestataires de la période 1968 auxquels Michel a participé (Gobille, 2008). Largement partagée par sa femme, cette disposition le pousse à s'interroger sur les logiques sociales et politiques de la reproduction de la pauvreté :

J'avais remarqué comment c'était organisé, c'est-à-dire qu'il y avait des clubs de gens corrompus. [...] Je les ai vus chercher différents moyens pour faire tomber des gens. Ça, cela m'a... ça, c'est un déclic pour moi. [...] J'ai commencé à 
développer une première réflexion politique [sur les politiques de développement], un premier cadre de réflexion, des priorités, des choses [sur] les relations villes-campagnes, les villes secondaires, et puis le développement participatif.

Son aversion pour le directivisme institutionnel (" J'étais un peu vacciné contre l'idéologie institutionnelle ») renforce alors sa remise en question progressive des principes du développement fondé sur une division verticale et «top-down » de la distribution de l'aide économique qui prête peu d'attention à la manière dont celle-ci devrait, selon lui, permettre à ces groupes sociaux de sortir de l'enfermement d'un système qui leur est défavorable. Ainsi, après une période d'apprentissage et d'adaptation, il entreprend un long travail de persuasion auprès de la Banque mondiale pour contester la démarche consistant à imposer aux populations locales les projets et conditions de développement établis par ces décideurs. En refusant l'aide charitable, (« j’ai pris cette distance par rapport à cette victimisation du pauvre qu'on a beaucoup dans les milieux chrétiens ») tout comme la logique de l'investissement économique sans consultation et implication des populations locales (Banque mondiale), il plaide pour une approche visant à lever les obstacles à la réalisation de projets élaborés par celles-ci :

J'ai pu modifier un peu le cours de la Banque mondiale, parce qu'elle a toujours besoin de savoir [à l'avance] qu'est-ce qui va être fait et combien ça coûte. Et là je disais que l'important c'est justement d'inverser la priorité. J'avais beaucoup travaillé pour qu'on accepte le principe que les gens pauvres participent financièrement. [...] Et puis j'ai compris que l'aide c'était pas donner, c'était faciliter, c'était enlever les empêchements, par exemple dans l'accès au crédit.

Cet apprentissage et ses réflexions sur le terrain se poursuivent tout au long de sa carrière, notamment à Madagascar où il collabore étroitement à un programme de lutte contre la corruption avec la Ministre de la Justice, puis en Asie. Ces expériences l'amènent à approfondir sa connaissance des logiques culturelles et locales des relations de pouvoir, sollicitant son ouverture aux autres croyances religieuses et sa compréhension du rôle des traditions indigènes dans le maintien et la justification de systèmes sociaux dont il déplore les iniquités :

J'ai lu le Coran, et comme j'allais parfois au Sri Lanka pour la formation, j'ai lu des textes bouddhiques aussi. Et quand j'étais au Pakistan, j'ai lu le Veda, donc j'ai essayé... mais c'était aussi par intérêt personnel, un peu. C'était dans cette période plus agnostique et moins liée à la tradition chrétienne.

Ces circonstances réveillent alors son intérêt et sa compétence pour l'étude des textes bibliques que l'on pouvait penser définitivement évanouis, et qu'il mobilise pour traiter des questions politiques, en particulier permettre des réponses collectives à donner à la violence :

C'était un petit peu regarder autour ce qui se disait dans d'autres traditions. Ça... cela m'a un petit peu conforté dans mon côté christianisme. [...] Par exemple, dans la façon de révéler la violence. Les gens disent toujours, tendre l'autre joue parce qu'on est très gentil, et tellement gentil que... et en fait, c'est 
simplement dire : « Est-ce que tu veux vraiment me gifler? Tu m'as giflé là ! Est-

ce que tu persistes dans ta violence envers moi ? ».

Ces réflexions le conduisent à remettre en question l'orientation instrumentale des politiques menées dans la régulation des relations locales, nationales et internationales reposant essentiellement sur des leviers économiques, et à défendre la pertinence du respect des valeurs en politique. Au cours de séminaires dispensés lors de ses séjours en Inde, à Madagascar ou encore au Rwanda où il est reçu en tant que haut fonctionnaire de la Confédération, Michel développe et argumente la pertinence de l'« éthique en politique ». Une telle approche n'a rien d'anodin dans la mesure où les institutions qu'il fréquente tout au long de sa carrière professionnelle ne fournissent pas d'incitations particulières et de ressources spécifiques pour ce type de réflexion. Or, au terme de sa carrière, il publie un ouvrage d'éthique politique qui, aujourd'hui, fait référence en la matière pour la formation en relations internationales, notamment à Genève où il enseigne dans une école de hauts fonctionnaires, et plus largement dans le champ intellectuel de la réflexion éthique.

Le parcours de Michel montre ainsi comment le politique en vient à investir la sphère religieuse, mais aussi comment le religieux investit le politique dans le cadre de son activité professionnelle. On voit notamment la manière dont certaines dispositions incorporées avant l'entrée dans un milieu professionnel peuvent conduire à remettre en question les principes régissant ce milieu. La comparaison avec un second parcours exposera comment l'accès à d'autres types de responsabilités et de ressources mobilisables contribue aux choix réalisés dans la carrière professionnelle, syndicale cette fois. Le parcours de Pierre, qui passe de la jeunesse rurale chrétienne au syndicat puis aux Ressources humaines, montre en effet un tout autre type de faisceau de déterminations dans la manière d'endosser son rôle professionnel.

\section{Pierre : de la Jeunesse rurale catholique à la Direction des ressources humaines}

Pierre, né en 1949, grandit dans un village catholique rural « entièrement contrôlé par le curé et ses gens ». Son père, "domestique de campagne », est issu d'une famille de 16 enfants qui « était à l'assistance », financièrement pris en charge par la «bourgeoisie $e^{4}$ » : « Mes grands-parents, ils n'avaient pas un mot à dire à l'assemblée communale, ni mon père, parce que c'était tacitement dit que c'était "grâce à nous, les bourgeois paysans de cette commune, que vous êtes là” ». Pierre garde le souvenir d'un village marqué par "l'hypocrisie fondamentale» des échanges cordiaux du village qui masquent des rapports sociaux les reléguant, lui et sa famille, à un statut subalterne. Il explique ainsi la manière dont sa mère, sans formation, est rejetée par sa famille «parce que quand

${ }^{4}$ Appellation officielle des habitants originaires du lieu (du « bourg »), dépositaires de l'autorité locale et d'une certaine fortune, que Pierre qualifie de "groupes sociaux très importants ». 
même ça ne se fait pas d'épouser un domestique ", avant d'être brutalement licenciée « de la poste du village ». À l'école, « c'était le contrôle et la sévérité, la baguette. [...] On était deux dans notre classe où on était des boucs émissaires, parce que fils de domestique, etc. Pour moi c'est clair !» Il aligne les exemples d'une " souffrance perpétuelle, d'une domination, d'une exploitation, d'une injustice » qui l'animent émotionnellement dès son plus jeune âge : « J'étais un gamin toujours un peu révolté ! ».

Durant toute son enfance, il fréquente les scouts de son village, puis la Jeunesse rurale catholique (JRC) de sa région. Pierre les décrit rétrospectivement comme des espaces de sociabilité et d'entre-soi juvénile où les rapports de domination au village sont suspendus : « Les enfants du village, on était beaucoup ensemble pour jouer, donc les enfants cassaient le système traditionnel, relationnel, selon lequel il devait y avoir des séparations ». Ce qu'il dit apprécier avant tout dans ces groupes, c'est le « travail collectif », décrivant ces activités comme un domaine de sa vie où les rapports de domination seraient inversés en sa faveur : « Moi j'étais un leader, très rapidement. J'avais des prises de pouvoir».

Sa socialisation à la JRC se déroule à un moment où l'Action catholique rurale est traversée par un large mouvement de fond. Dès l'après-guerre, ses théoriciens - admettant l'impossible retour vers le modèle de la chrétienté catholique - abandonnent la stratégie consistant à maintenir les jeunes catholiques ruraux à l'écart de la modernité et du libéralisme. Désormais, la JRC doit former des militants qui, à travers des méthodes d'enquête sur la réalité qui les entoure, soient capables de transformer le monde pour « refaire [les] frères chrétiens» (Abiven et Calvez, 2004). Cette transformation prend une tournure particulière lors du Concile Vatican II qui consacre aux laïcs une place de premier ordre dans l'Église en organisant, par exemple, une journée d'apostolat des laïcs (i.e. l'organisation et l'animation de la messe par eux) et qui éveille, chez de nombreux animateurs de l'Action catholique, une aspiration à ouvrir la jeunesse au monde. La JRC fréquentée par Pierre constitue alors un lieu de découverte et d'assimilation d'outils critiques susceptibles d'être retournés contre l'institution religieuse, phénomène également observé par L. Mathieu en France (Mathieu, 2010). Pierre participe à ce mouvement en suivant les formations de responsable de la JRC, participant aux discussions sur l'engagement chrétien dans le monde et prenant progressivement la parole en public dans l'église lorsque l'occasion lui en est donnée :

On critiquait très fortement la hiérarchie et le conservatisme de l'Église. [...] On a passé beaucoup de temps et de week-ends de formation, voire des semaines de formation sur des thèmes... qui étaient liés à l'engagement, nouvelle façon de lire l'Évangile, de s'engager, ou comment changer le monde et comment je vais m'y prendre.

Tout au long de son récit, on retrouve dans le discours de Pierre l'expression d'une colère contre l'assignation des individus à des rôles subalternes qui l'incite à la prise de parole et développe un sentiment de révolte, sans doute entretenu par l'identification à des figures subversives découvertes lors des rencontres de la JRC : «J'étais révolté 
dans ce village parce que je voyais plein de trucs qui ne jouaient pas. Et c'est probablement ça qui a déclenché la nécessité pour moi d'avoir des leaders ou des référentiels. Et à ce moment-là c'était l'Église de Jésus-Christ ».

Durant cette période, Pierre effectue un apprentissage d'employé de commerce. Dans son travail, il se décrit comme quelqu'un qui fait preuve d'initiative, et il évoque positivement le patron de la petite entreprise où il travaille comme étant un entrepreneur astucieux : « Il était à la fois le constructeur des monte-charge 'Gendre', un brevet international unique au monde, reconnu par les États-Unis où il a fait une succursale. C'était impressionnant ! » Cette manière de percevoir son patron est révélatrice d'un schème de perception d'opposition aux "gros » - aux dirigeants du village, de l'Église, des grandes entreprises comme celle où travaille son père pour un salaire qu'il juge insatisfaisant - par des « petits » - les enfants du village, la figure du Christ, le petit patron capables de leur résister par leur génie.

Parallèlement à son travail, l'intériorisation de dispositions critiques dans le cadre de la JRC s'accompagne d'une aspiration à acquérir une autonomie matérielle et intellectuelle dont son milieu familial semble dépourvu : « Moi j’ai toujours eu l'idée, autonomie, autonomie ! Donc c'était de partir le plus vite possible. [...] J'ai appris, moi, dans la JRC, à dire "tu dois faire ta propre analyse des choses" ». Il évoque, non sans fierté, l'étonnement de son père, lorsqu'il est ensuite recruté en tant que permanent à la JRC, juste après avoir terminé son apprentissage : « Mon père me disait "Pierre il ne travaille pas, et il gagne 600 Frs. par mois...” !».

Comme le montrent cependant Yohann Abiven et Eugène Calvez (2004), l'approche de l'Action catholique « a vite montré ses limites : elle ne fournissait pas de causes généralistes aux phénomènes étudiés, mais des solutions toutes-faites, adaptées peut-être à une situation isolée, mais plus inappropriée quand on pense le fonctionnement global d'une société développée. Les militants deviendront demandeurs de cadres théoriques pour la pensée ». Dans un contexte international et national marqué par la formation de groupes gauchistes, Pierre décide en effet, avec une dizaine d'amis de la JRC, de former en parallèle un groupe d'action politique d'inspiration marxiste par le biais d'échanges avec d'autres militants de la jeunesse étudiante chrétienne qui, entretemps, se sont politisés à gauche. Il décide également de devenir responsable au Mouvement international de la Jeunesse agricole et rurale catholique (MIJARC), une organisation de développement rural catholique qui l'amène à voyager en Afrique et en Amérique latine. La dimension politique de son militantisme se substitue progressivement à sa dimension religieuse, sans toutefois provoquer de « crise » majeure : « Je devais être officiellement un militant de l'Action catholique, mais j'avais déjà à l'époque quitté l'Église, enfin dans ma tête ». Devant le constat de ce qu'il considère être son inefficacité, et suite à de multiples échanges avec des militants marxistes, Pierre abandonne son poste au MIJARC et se distancie définitivement de l'Église et de la foi. De retour dans sa région natale, père de deux enfants et en couple avec une ancienne militante 
de la JRC, il reprend un travail de comptable dans une entreprise de la région et s'investit dans le groupe militant d'inspiration marxiste.

Composé d'une petite dizaine de militants, le groupe peine à recruter de nouveaux membres et à exercer une réelle influence sur les ouvriers et paysans de la région. Le groupe décide alors de fusionner avec une organisation marxiste-léniniste interrégionale qui traverse un processus de division hiérarchique et tolère de moins en moins les écarts de ses membres à ses thèses politiques 5 . Si l'intégration dans cette organisation fournit à Pierre un cadre théorique d'explication convaincant des inégalités sociales, elle bute contre une disposition anti-remise de soi qu'il a incorporée au cours de ses engagements passés, à la JRC et au MIJARC, où il a exercé des tâches valorisant l'autonomie réflexive et l'initiative individuelle : « Et tout d'un coup, il y avait une ligne qui changeait, et il fallait faire l'autocritique. Et si tu ne la faisais pas, tu étais convoqué par les chefs ». Alors que cet engagement l'épuise et perd de son sens à ses yeux, un ancien permanent de la JRC le contacte pour occuper un poste de secrétaire dans un syndicat inspiré du syndicalisme autogestionnaire français. Pierre endosse alors ce rôle sur le mode d'une professionnalisation de son engagement militant mieux ajustée à sa disposition « anti-remise de soi » et valorisant ses ressources militantes :

[Au syndicat] j'ai pu prendre de l'autonomie et mon pouvoir. C'est d'ailleurs pour cela que j'ai quitté [l'extrême-gauche], pour pouvoir concrétiser les revendications des travailleurs, transformer leur statut, les conventions collectives.

Cette transition clôt une période de militantisme « dans la marge » de l'espace militant, et aboutit également à la séparation du couple : "J'avais une image de Marie comme une image idéale pour moi qui n'était plus là ». Durant les vingt années passées au syndicat, Pierre tire une grande satisfaction de son travail qui lui apporte une respectabilité et du crédit personnel : «J'étais connu comme le loup blanc. J'avais commencé à entrer partout ». L'accumulation de ressources militantes semble faciliter son apprentissage du métier syndical. La satisfaction d'acquérir, selon lui, une réputation de négociateur semble l'entraîner dans un processus que C. Leclercq décrit comme un renforcement de l'estime de soi favorable à l' affranchissement des schèmes doctrinaux transmit par des groupes et dirigeants politiques tutélaires, ainsi qu'à l'ouverture des «perspectives d'autonomisation individuelle » et aux « contre-définitions » du travail politique (Leclercq, $2011: 134$ ) : « Moi j'étais leader numéro un, et cela me posait problème qu'on prenne ces gens [patrons, politiciens, etc.] pour des cons. J'avais besoin d'avoir en face de moi un partenaire pour conclure quelque chose ». Cette posture d'affranchissement permet à Pierre de se présenter comme un militant lucide refusant

\footnotetext{
${ }^{5}$ Dans sa thèse sur l'anti-impérialisme en Suisse, I'historien Nuno Pereira explique que la structure organisationnelle de ce groupe connaît « un processus de centralisation qui la conduit à devenir une formation très rigide, tant sur le plan doctrinal qu'organisationnel » (Pereira, $2015: 156)$
} 
d'appliquer des principes politiques qui lui ôteraient des prérogatives d'action concrètes, prenant ainsi ses distances vis-à-vis d'une " vulgate » marxiste :

On me disait: «Vous faites beaucoup de compromis ». Oui, parce que c'est le mot à l'époque, et moi je pense que ce n'était pas des compromis au sens vrai. C'était des accords. C'était des accords qui servaient les intérêts de chacun.

Suite à des difficultés internes, le syndicat fusionne par la suite dans une organisation syndicale concurrente. Pierre se retrouve alors dans une organisation syndicale aux effectifs salariés importants et dotée d'une structure organisationnelle hiérarchisée et centralisée. À partir des années 1990, ce façonnage organisationnel se caractérise par une professionnalisation croissante du métier syndical avec le recrutement de diplômés universitaires sans être nécessairement doté d'une expérience militante (Guillaume, 2018). Vécu par certains permanents expérimentés comme un discrédit des ressources militantes et une minorisation de "la dimension "sacrificielle" de l'engagement syndical » (Guillaume et Pochic, 2009: 55), ce changement est perçu par Michel comme une déviance bureaucratique du travail syndical : « [Quand] j'étais dans ce syndicat, alors là les compromis... j'avais presque honte pour ma base. [...] Et puis c'est vrai que tous les autres étaient des universitaires. Je n'ai rien contre les universitaires, mais ¡'avais beaucoup de peine ».

Ce contexte le pousse à passer à une autre étape de sa carrière professionnelle. La cinquantaine, en couple avec une femme peu politisée («pour Vivianne, militer c'est pas... elle ne sait pas ce que cela veut dire »), Pierre démissionne du syndicat. Il décroche aussitôt un poste de directeur des ressources humaines (DRH) en milieu hospitalier, un emploi qui sollicite à plus d'un titre ses ressources et compétences, tout en étant ajusté à son patrimoine dispositionnel (compétences et goûts pour la gestion du collectif et le leadership, goût pour l'autonomie...). Cet espace professionnel active et renforce son appétence pour la conduite de négociations et la gestion des relations sociales, revendiquant comme d'autres DRH (Surdez et al. 2016) l'autonomie de sa position à l'égard de la direction. La fréquentation de cet univers professionnel agit alors comme une socialisation de renforcement, en valorisant les qualités associées à l'entrepreneur et au leader capable de se faire entendre et respecter. L'univers de la gestion des ressources humaines est occupé en partie par des individus dont l'ascension professionnelle a été rendue possible par l'accès à la DRH en gravissant un à un les échelons de l'entreprise, ce qui est source d'un sentiment « de gratitude envers le monde de l'entreprise » (Surdez et al., 2016). Après cinq ans à la DRH, Pierre se dirige vers le consulting et dispense des cours de gestion des ressources humaines dans une haute école, activités qu'il exerce jusqu'à sa retraite.

Cette stratégie de requalification « de l'autre côté de la barrière " grâce aux ressources accumulées dans l'action militante constitue « une improbable transgression aux traditions syndicales » (Rimbert, 2006: 21), même si le secteur public hospitalier est peu sujet aux licenciements de masse et à la recherche de profit. Cette transgression ne va pas sans exercer une « contrainte de sens » (Tissot, 2005 : 15) qui pousse Pierre à porter 
un discours visant à mettre son travail en adéquation avec son engagement militant, lui qui entretient des amitiés de longue date avec des militants et qui, contrairement à plusieurs d'entre eux, accède à une position rémunératrice lui donnant accès à la propriété individuelle tout en réduisant son temps de travail ("C'était aussi pour ma vie de couple, parce que j'ai vite compris après deux divorces que tu dois cultiver »). À divers moments de l'entretien, il associe un caractère syndical à son travail, qu'il dit centré sur l'écoute du personnel et la mise sur pied de dispositifs participatifs. Tout en rejetant les schèmes doctrinaux du gauchisme et ayant accédé à une profession qui le place en porte-à-faux à l'égard de ceux-ci (Tissot, 2005: 13), il se présente comme un DRH en position de défendre réellement les intérêts des employés face à la direction :

Des fois on me dit : "Comment tu peux faire RH dans une boîte ? ». Mais je travaille avec la nettoyeuse autant qu'avec le patron ! Je pense que là je fais plus de travail syndical qu'à l'extérieur. [...] [Les employés] me mettaient au défi, sans arrêt, «parce que tu es engagé pour faire des économies sur le personnel», sans arrêt. Et moi j'ai dit qu'on n'a pas fait d'économie, mais qu'on a transformé là, on a roulé dans la farine [la manager et cheffe de projet adjointe à la direction] avec les effectifs qui nous fallait pour conserver le personnel.

Durant l'entretien, il exhibe plusieurs livres de sociologie et psychologie des organisations qu'il a soigneusement surlignés et annotés. En se référant à ces ouvrages et à son expérience, il diagnostique une transformation profonde des rapports au travail qui le place en théoricien et spécialiste des ressources humaines distancié des pratiques, croyances et normes instituées dans l'univers syndical et professionnel :

On a eu des débats théoriques autour d'un philosophe [...] qui est parti très fort pour démonter tout le système de subordination dans l'entreprise. Mais il ne se rend pas compte que dans ce système, ceux qu'il appelle les « soumis » trouvent du plaisir ou ont certains intérêts à l'être. [...] Et c'est nouveau ça ! Et ça fout en l'air toute l'histoire de la force des syndicats.

Cet investissement intellectuel indique le travail continu de mise en cohérence qui accompagne sa bifurcation professionnelle. Résumé en quelques phrases, le parcours de Pierre se caractérise par l'apprentissage de compétences organisationnelles et l'incorporation d'une disposition à « l'anti-remise de soi » au cours de sa socialisation militante, à la JRC puis au syndicat. La rencontre avec des contextes militants changeants, à savoir des groupes gauchistes et syndicaux traversant un processus de centralisation et de hiérarchisation contraire à son appétence pour l'autonomie individuelle, le pousse à rejeter ces derniers et à adopter des contre-définitions du travail politique en accord avec son orientation vers les ressources humaines. Autrement dit, l'entrée de Pierre dans les ressources humaines s'accompagne d'une révision des contenus idéologique intériorisés durant son engagement gauchiste et syndical, lui permettant de reconvertir un savoir-faire militant dans les RH sans éprouver en même temps le sentiment de trahir ses engagements militants passé. 


\section{Conclusion}

On a démontré empiriquement que la marge de jeu inhérente à l'endossement des rôles institués est largement déterminée par l'intériorisation de dispositions, de croyances et de vision spécifique du monde, et par leurs révisions en fonction des contextes organisationnels tout au long du parcours de vie (Lahire, 2013). En somme, la structure organisationnelle et les normes instituées qui encadrent l'activité professionnelle ne peuvent rendre raison à elles seules de la manière dont les individus investissent leur rôle professionnel.

On voit bien dans ces deux parcours les incidences des différents contextes de socialisation transformant, notamment, la croyance aux biens du salut dans l'au-delà en appétence pour la défense de certaines valeurs (la transmission d'une foi et de valeurs qui engagent des pratiques telles que l'engagement solidaire, la prise de parole et la participation collective transférables à l'action militante et professionnelle). Inversement, le comportement des individus ne se réduit pas à l'importation et à la conversion de dispositions dans l'univers professionnel, car celui-ci implique d'endosser des rôles institués, et trop « s'en écarter c'est s'exposer à la réprobation et à la disqualification » (Lefebvre, $2011: 221$ ).

Dire cela, c'est rappeler que les acteurs n'importent pas des dispositions religieuses et militantes dans la sphère professionnelle, mais que ces dispositions sont sollicitées, mises en veille ou refaçonnées par des contextes plus ou moins codifiés et normés, et que leur mobilisation s'accompagne d'un effort de mise en cohérence du passé avec le présent par l'acteur à travers un travail introspectif sur sa biographie. L'analyse de ces deux portraits attire l'attention sur la propension de certains militants à investir des activités professionnelles encadrées par des normes relativement souples pouvant faire l'objet d'appropriations et d'interprétations très différentes.

\section{Bibliographie}

Abiven Y., Calvez E. (2004), «Les avatars de l'identité catholique: les militants de la ruralité (1929-2000)», in B. WACHE (dir.), Militants catholiques de l'Ouest, Rennes, Presses universitaires de Rennes, p. 67-103.

DENAVE S. (2015), Reconstruire sa vie professionnelle: sociologie des bifurcations biographiques, Paris, Presses Universitaires de France.

Fillieule O., Beroud S., MASClet C., Sommier I. (2018), Changer le monde, changer sa vie. Enquête sur les militantes et militants des années 1968 en France, Arles, Actes Sud.

Fouilloux É. (2000), «Les voies incertaines de l’œcuménisme (1959-1999)», Vingtième Siècle. Revue d'histoire, vol. 66, n 1, p. 133-145. 
Gobille B. (2008). « La vocation d'hétérodoxie », in D. Damamme, B. Gobille, F. Matonti, B. PudAL, Mai-Juin 68, Éditions de l’Atelier, Yvry, p. 274-294.

Guillaume C. (2018), Syndiquées : défendre les intérêts des femmes au travail, Paris, Presses de SciencesPo.

LAGROYE J. (2006), La vérité dans l'Église catholique: contestations et restauration d'un régime d'autorité, Paris, Belin.

LAHIRE B. (dir.) (2011), Ce qu'ils vivent, ce qu'ils écrivent : mises en scène littéraires du social et expériences socialisatrices des écrivains, Paris, Archives contemporaines.

LAHIRE B. (2013), « La fabrication sociale des individus : cadres, modalités, temps et effets de socialisation ", in Dans les plis singuliers du social. individus, institutions, socialisations, Paris, La Découverte, p. 115-132.

LECLERCQ C. (2011), « Engagement et construction de soi. La carrière d'émancipation d'un permanent communiste », Sociétés contemporaines, vol. 84, $n^{\circ}$ 4, p. 127-149.

LEFEBVRE R. (2011), "Se conformer à son rôle. Les ressorts de l'intériorisation institutionnelle », in J. LAgroye, M. OfFerle (dir.), Sociologie de l'institution, Paris, Belin, p. 219-247.

MATHIEU L. (2010), «Les ressorts sociaux de l'indignation militante. L'engagement au sein d'un collectif départemental du Réseau éducation sans frontière », Sociologie, vol. $1, n^{\circ} 3$, p. 303-318.

PAGis J. (2011), «Incidences biographiques du militantisme en Mai 68 », Sociétés contemporaines, vol. $84, \mathrm{n}^{\circ} 4$, p. 25-51.

Pelletier D., SChlegel, J.-L. (2012), À la gauche du Christ. Les chrétiens de gauche en France de 1945 à nos jours, Paris, Éditions du Seuil.

Pelletier D. (2002), La crise catholique. Religion, société, politique en France (1965-1978), Paris, Payot et Rivages.

PEREIRA N. (2015), Anti-impérialisme et nouvelle gauche radicale dans la Suisse des années 68 , thèse de doctorat en science politique, Université de Lausanne.

PLANZI L. (2011), « Le calme plat avant la tempête dans le clergé romand : le premier ébranlement dans le recrutement, la formation et le statut (1945-1960)", Schweizerische Zeitschrift für Religions- und Kulturgeschichte, vol. 105, p. 325-338.

Ponsard N., Rousseau S. (2009), « Pour une histoire des lectures militantes au XX ${ }^{\mathrm{e}}$ siècle en France », Siècles, vol. 29, p. 3-11.

Rimbert P. (2006), «"De l'autre côté de la barrière”. La reconversion "patronale” de syndicalistes ouvriers dans les années 1990 », in S. TissoT, C. GAUBERT, M.-H. LECHIEN (dir.), Reconversions militantes, Limoge, Presses Universitaires de Limoges, p. 21-49. 
RIST G. (2013), Le développement: histoire d'une croyance occidentale, Paris, Presses de la Fondation nationale des sciences politiques.

Sainsaulieu I., Surdez, M. (dir.) (2012), Sens politiques du travail, Paris, Armand Colin (Recherches).

Simeant J. (2001), « Entrer, rester en humanitaire. Des fondateurs de Médecins sans frontières aux membres actuels des ONG médicales françaises », Revue française de science politique, vol. 51, $\mathrm{n}^{\circ} 1-2$, p. 47-72.

SimEANT J. (2009), "Socialisation catholique et biens de salut dans quatre ONG humanitaires françaises », Le Mouvement Social, vol. 227, n 2, p. 101-122.

Surdez M., Zufferey E., Sainsaulieu I., Plomb F., Pog F. (2016), L'enracinement professionnel des opinions politiques: enquête auprès d'agriculteurs, d'ingénieurs et de directeurs de ressources humaines exercant en Suisse, Zurich, Seismo.

Tissot S. (2005), « Reconversions dans la politique de la ville : l'engagement pour les “quartiers" », Politix, vol. 70, nº 2, p. 71-88.

Voegtli M. (2004), « Du Jeu dans le Je : ruptures biographiques et travail de mise en cohérence », Lien social et Politiques, nº 51, p. 145-158. 